Rev. Int. Contam. Ambie. 37, 187-199, 2021

https://doi.org/10.20937/RICA.53779

\title{
DEGRADACIÓN AMBIENTAL ANTRÓPICA DE LAS BARRANCAS EL CONDE Y MALINALLI DE LA CIUDAD DE PUEBLA, MÉXICO
}

Anthropic environmental degradation of El Conde and Malinalli ravines from Puebla city, Mexico

\author{
Víctor GUTIÉRREZ PACHECO* y Sonia Emilia SILVA GÓMEZ
}

Posgrado en Ciencias Ambientales, Instituto de Ciencias, Benemérita Universidad Autónoma de Puebla, Edif. 103 D (planta baja), Col. Jardines de San Manuel, 72570 Puebla, Puebla, México.

*Autor para correspondencia: v.guty@yahoo.com.mx

(Recibido: octubre de 2019; aceptado: junio de 2020)

Palabras clave: asentamientos humanos irregulares, biodiversidad, contaminación.

\section{RESUMEN}

Un porcentaje de los desechos generados en la ciudad de Puebla se elimina sin control en el sistema de barrancas de la urbe. Por esta causa y debido a la presencia de asentamientos humanos irregulares, dichas barrancas sufren procesos de degradación que ponen en riesgo su continuidad y con ello los servicios ecosistémicos que proporcionan a la ciudad. Tal situación se exhibe en el estado en que se encuentran las barrancas El Conde y Malinalli, el cual ejemplifica lo que ocurre en el sistema urbano de barrancas. La evaluación se hizo a través de los índices de Margalef y de Pielou, así como de un conjunto de nueve indicadores que miden diversos componentes de las barrancas. Los resultados indican que el grado de diversidad de especies en ambas barrancas es medio a alto. La barranca El Conde presenta un estado ecosistémico pobre, que es el segundo nivel más bajo en una escala de cinco niveles de calidad y tiene que ver con el impacto generado por la acción antrópica, mientras que la barranca Malinalli exhibe un nivel de calidad excelente. La diversidad de especies, en tanto indicador de alta calidad ecológica y criterio para la preservación de los ecosistemas, sustenta la propuesta de recuperar y conservar ambas barrancas, acción urgente para el caso de la barranca El Conde por el impacto antrópico que exhibe.

Key words: biodiversity, irregular human settlements, pollution.

\begin{abstract}
A percentage of the waste generated in Puebla city is disposed of without control in the city's ravine system. Due to this, and to the presence of irregular human settlements, the ravines suffer degradation processes that put their continuity at risk, endangering the ecosystem services they provide to the city. That situation is shown in the condition of the El Conde and Malinalli ravines, which exemplify what happens in the urban system of ravines. The evaluation was done through the Margalef and Pielou indexes and a set of nine indicators that measure various components of the ravines. The results indicate that both ravines are diverse regarding the species category, from a medium to a high level. El Conde ravine presents a poor ecosystem status, which is the second lowest level on a five-level quality scale and is related to the impact generated by
\end{abstract}


anthropic action, while Malinalli ravine exhibits an excellent quality level. The diversity of species, as an indicator of high ecological quality and criteria for the preservation of ecosystems, supports the proposal to recover and preserve both ravines, an urgent action in the case of El Conde due to the anthropic impact it is undergoing.

\section{INTRODUCCIÓN}

Al igual que muchas ciudades de México, Latinoamérica y el mundo, la ciudad de Puebla experimentó un crecimiento poblacional espectacular en la segunda mitad del siglo pasado y las casi dos décadas del presente, pasando de 234603 habitantes en 1950 (año en que se acentúa la industrialización de la ciudad) a 1576258 en 2015 (INEGI 1994, 2015). Este crecimiento originó que la mancha urbana pasara de ocupar un $4.07 \%$ respecto del territorio total municipal en e 1970 a un $39.86 \%$ en 2005 (Ayuntamiento de la Ciudad de Puebla 2008) y que se generaran procesos de conurbación con localidades cercanas, tanto del mismo estado de Puebla como del estado vecino de Tlaxcala. Esto ha derivado en que ahora se le considere, para efectos de planeación y de instrumentación de políticas públicas, como la Zona Metropolitana Puebla-Tlaxcala con una población contabilizada para 2015 de 2941988 habitantes (CONAPO 2018).

Desde la época colonial, la ciudad de Puebla se distinguió como una de las más importantes del Virreinato de la Nueva España debido, entre otros factores, a su preeminencia económica y actividad industrial, la cual nunca ha abandonado a pesar de vaivenes históricos como la Revolución mexicana, lo que se refleja en que actualmente se asientan en ella 21 parques industriales con actividades que abarcan rubros como el alimenticio, textil, químico, petroquímico, automotriz, papelero, de bebidas, hierro y acero, farmacéutico, curtido de pieles, metal-mecánico, siderúrgico y de servicios, entre otros (Gamboa 2010, IMTA-CONAGUA 2013, SIMPPI 2018).

Si bien es cierto que la actividad industrial es generadora de riqueza, también lo es de una gran cantidad de desechos sólidos y de aguas residuales con altos contenidos de sustancias tóxicas que se vierten sin tratamiento en las barrancas y cuerpos de agua naturales de la ciudad, lo que ha llevado a que el río Atoyac y la presa de Valsequillo (que son los cuerpos de agua a donde se derivan todos los cauces naturales y artificiales de la ciudad) presenten altos niveles de contaminación, señalándose al primero como uno de los más contaminados del país (IMTACONAGUA 2013).
Estos dos fenómenos urbanos, el crecimiento poblacional y la actividad industrial, han provocado configuraciones y relaciones inéditas entre los habitantes y su medio biofísico, lo que ha generado una gran demanda de recursos y energía, así como la descarga y eliminación - sin precedentes históricos - de materiales degradados y energía disipada hacia el entorno.

El crecimiento de la mancha urbana ha provocado el encarecimiento de la tierra y la disminución de su oferta, lo que ha orillado a personas de los estratos sociales más desprotegidos a buscar una alternativa de vivienda y la han encontrado en "terrenos de nadie", es decir, sitios que por su fisiografía no son aptos para uso habitacional en las barrancas de la ciudad. Todo ello inscrito en un modelo de urbanización de la pobreza que se caracteriza entre otras cosas por: $a$ ) déficit de servicios de base, $b$ ) habitación informal, c) vulnerabilidad económica por desastres naturales y problemas ambientales y $d$ ) desigualdad creciente al interior de las ciudades (López 2005).

Aunado a la ocupación de las barrancas con fines habitacionales, se da la eliminación, por dichas personas y por otros particulares como empresas constructoras, de residuos sólidos y aguas residuales sin tratamiento hacia el sistema de barrancas de la ciudad, utilizándolo como canal de desagüe y vertedero de basura al aire libre. Esto, a pesar de la existencia de sistemas municipales de drenaje y de recolección de residuos sólidos, que se muestran insuficientes, y de la normatividad vigente en materia de eliminación de residuos sólidos urbanos y de manejo especial (Ley General para la Prevención y Gestión Integral de los Residuos [SEMARNAT 2003]), y de disposición de aguas residuales hacia los canales naturales [NOM001-SEMARNAT-2006] (SEMARNAT 1997).

Estas acciones de contaminación, en conjunto con el impacto que genera la vida en las barrancas, ha propiciado que el sistema de barrancas presente visible degradación y sea observado como un elemento negativo para la ciudad, en tanto se considera que afecta a los vecindarios donde se ubican por los malos olores generados, la fauna feral y su apariencia sucia, entre otros elementos (Silva et al. 2014).

Las barrancas de la ciudad de Puebla, originadas por vulcanismo y por los escurrimientos pluviales de 
la Malinche (IMTA-CONAGUA 2013) son fracciones de terreno de forma discernible que presentan un cambio de elevación de dos metros, como mínimo, entre el punto más alto y el más bajo; que pueden presentar cubierta vegetal y que tienen o han tenido en algún momento del año, corrientes de agua (City of Toronto 2018).

De acuerdo con el diccionario geomorfológico del IG-UNAM (1989) una barranca es una "forma lineal negativa del relieve, estrecha, con laderas abruptas [...] En longitud llega a alcanzar algunos kilómetros, y en anchura y profundidad, algunas decenas de metros [...] se forman generalmente en rocas incoherentes o fácilmente erosionables, como los loess, depósitos piroclásticos y conglomerados, por escurrimiento de temporada de las aguas pluviales y nivales" (p. 44).

Las barrancas cumplen múltiples funciones ecosistémicas para la urbe, como ser áreas de amortiguamiento por sus barreras de árboles, de refugio a la vida silvestre, de recarga de acuíferos, de regulación de inundaciones por azolves extraordinarios y de regulación del clima (Rivas 2001, Nowak et al. 2006), de tal manera que son elementos geomorfológicos muy importantes de los ciclos hidrológico y biogeoquímico y, al estar interrelacionadas, cualquier afectación a una zona determinada repercute sobre todo el sistema (SEDEMA 2000).

Sin embargo, a pesar de que las barrancas proporcionan servicios ecosistémicos (entendidos como los beneficios que la naturaleza aporta a la sociedad [FAO 2019]), han sido históricamente un componente marginal en el devenir de la ciudad. Incluso podría decirse que se han conceptualizado como un obstáculo para el progreso (Gutiérrez y Silva 2017).

Las barrancas merecen otra consideración dados sus servicios ecosistémicos, lo que debe implicar su recuperación y manejo. Para emprender acciones de recuperación y conservación del sistema barranca, es necesario conocer previamente lo que se tiene y en qué estado se encuentra (Sayre 2002). De ahí que se propone como paso inicial la evaluación del sistema considerando aspectos tanto de su biodiversidad y morfométricos, como de tipo socioeconómico. Esto en el entendido de que en la ciudad de Puebla hay pocos registros de trabajo académico o gubernamental que aborden este tema, pues la visión que permea hasta el momento en estos ámbitos es que las barrancas son espacios sin utilidad, lo que ha llevado a que algunas de ellas hayan sido entubadas y rellenadas para habilitar vialidades sobre ellas (Salgado 2018).

Asimismo, se considera que deben darse los primeros pasos hacia la sistematización de una metodología de evaluación sencilla - pero consistente y científicamente viable - cuyo objeto de estudio sean específicamente las barrancas urbanas, de tal manera que el trabajo no requiera de un personal altamente especializado y pueda realizarse en un tiempo razonablemente corto, de acuerdo con el espíritu de las evaluaciones ecológicas rápidas, ya que el deterioro ambiental al que están expuestas las barrancas así lo exige (Lovejoy 2002, Munné et al. 2003).

En este contexto son necesarios estudios como el presente, con el objeto de establecer nuevos fundamentos epistémicos que dejen atrás la visión utilitarista de la naturaleza y a partir de los cuales se desarrollen nuevos marcos teóricos y metodológicos que permitan el acercamiento a las barrancas de forma integral. Con ello podrían plantearse estrategias de conservación para las mismas por parte de los gestores, grupos sociales involucrados en temas de conservación, encargados de la toma de decisiones y la sociedad en general.

\section{MATERIALES Y MÉTODOS}

El sistema de barrancas de la ciudad de Puebla comprende una serie de rasgos geológicos de muchos kilómetros de longitud sin que hasta el momento exista un dato preciso de su extensión. Al recorrerse diversos tramos de dicho sistema se observan aguas contaminadas de origen doméstico e industrial, basura y asentamientos irregulares. Tal es el caso de barrancas como Alseseca, Los Álamos, Manzanilla, San Antonio, San Diego, Guadalupe y El Conde, todas dentro de la mancha urbana y cercanas a parques industriales como La Ciénega, 5 de Mayo, Puebla 2000 y Resurrección.

Partiendo de este escenario se consideró a la barranca El Conde para efectos de este trabajo de investigación, la cual se ubica al norte de la ciudad (Fig. 1) en las coordenadas $19^{\circ} 09^{\prime} 80^{\prime \prime} \mathrm{N}$ y $98^{\circ} 18^{\prime}$ 74 " O, con una longitud aproximada de $1700 \mathrm{~m} \mathrm{y}$ con un ancho que va desde los $30 \mathrm{~m}$ en algunos sitios hasta los $100 \mathrm{~m}$ en otros. En algunos lugares no se puede precisar la anchura, ya que los referentes se han perdido por la presencia de casas habitación. En cuanto a la profundidad, ésta varía en los diferentes sitios desde aproximadamente $60 \mathrm{~m}$ en las zonas más profundas hasta $0 \mathrm{~m}$ en aquellos sitios en que se realizaron obras de relleno, se construyeron casas en las orillas y se habilitó y pavimentó una calle.

El Conde recibe descargas de aguas residuales provenientes del parque industrial 5 de Mayo, con el que colinda, además de que es una de las barrancas menos abruptas y con mayores posibilidades de 


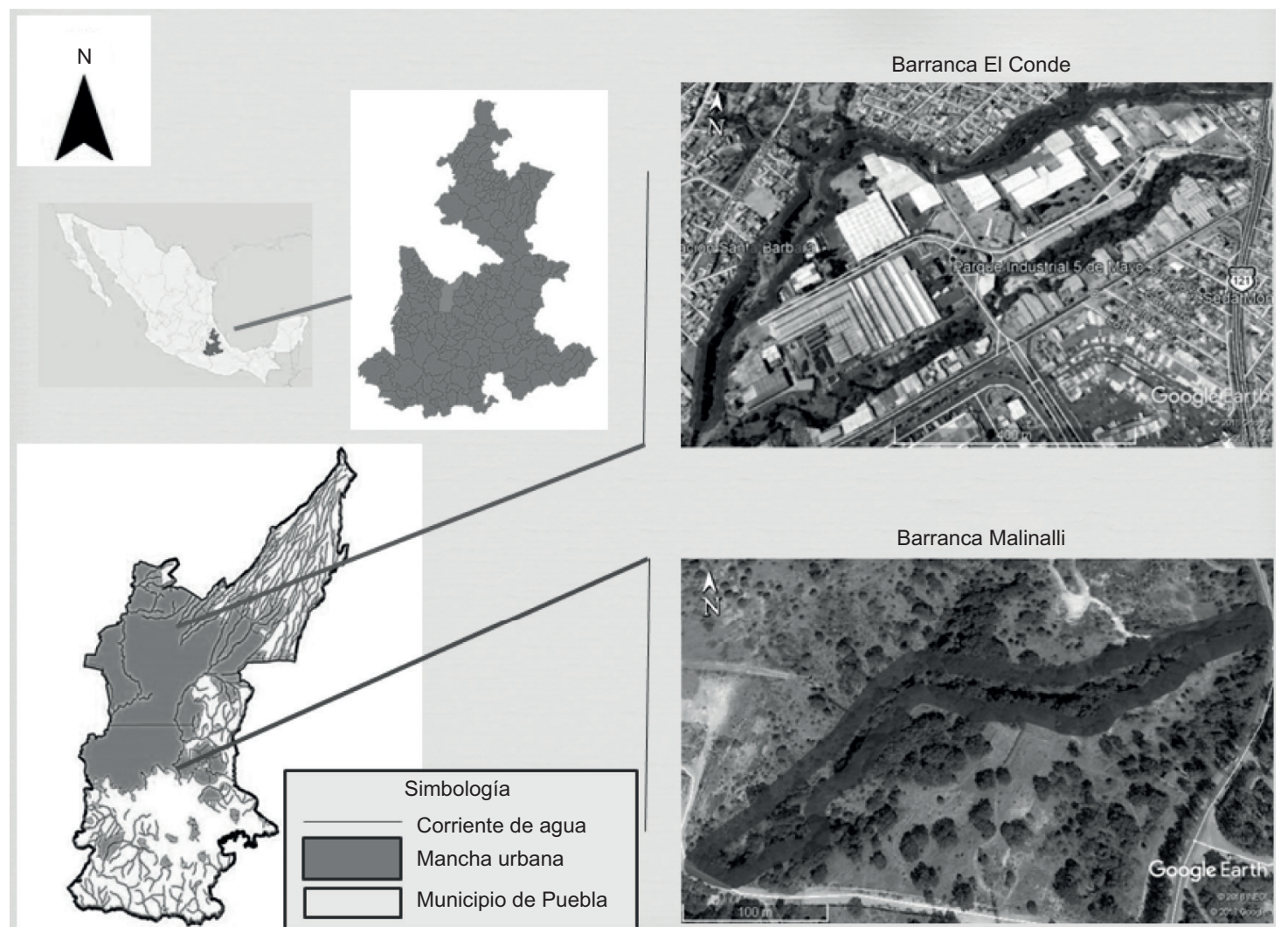

Fig. 1. Zona de estudio.

acceso. Un elemento adicional es que hay estudios, relativamente recientes, sobre los niveles de contaminantes presentes en las aguas que corren en su cauce (Díaz 2014, García 2014).

Para efectos comparativos, se evaluó también una barranca con características similares a El Conde en cuanto a tipo de ecosistema y grado de diversidad específica: la barranca Malinalli, que se encuentra hacia el sur de la ciudad (Fig. 1) en las coordenadas $18^{\circ} 93^{\prime} 93^{\prime \prime} \mathrm{N}$ y $98^{\circ} 13^{\prime} 68^{\prime \prime} \mathrm{O}$, con una longitud aproximada de $750 \mathrm{~m}$ y una profundidad que varía desde 1.30 hasta 15 o $20 \mathrm{~m}$. En cuanto a su anchura, también varía desde $8 \mathrm{~m}$ en los sitios más estrechos hasta $50 \mathrm{~m}$ en los más amplios. Malinalli tiene características ecosistémicas similares a las de otras barrancas de la periferia de la ciudad donde aún no llega plenamente la mancha urbana; por lo mismo, no presentan de manera evidente los impactos antrópicos que afectan a El Conde. Además, es accesible en tanto que se encuentra dentro de uno de los campus de la universidad estatal.

\section{Trabajo de campo y de gabinete}

Durante 2018 y 2019 se llevó a cabo una recolección selectiva de materiales botánicos cuando no fue posible la identificación in situ, mediante recorridos de campo que comprendieron el total del área de ambas barrancas,. Cada margen de la barranca El Conde se dividió en seis fracciones, para un total de 12 fracciones, mientras que la barranca Malinalli se dividió en tres fracciones en cada margen para un total de seis.

Conforme a los supuestos del método fitosociológico y a la escuela estadounidense de aproximación a la vegetación, se consideraron como especies de diagnóstico las especies leñosas (Alcaraz 1999). La identificación taxonómica se realizó mediante claves taxonómicas y bibliografía especializada (floras y monografías). Para la nomenclatura de los géneros y las especies se utilizó la base de datos The Plant List (TPL 2013).

Asimismo, se hicieron mediciones, tipificación y conteos de los elementos correspondientes a efecto de determinar porcentajes, números y tipos en gabinete, para alimentar los indicadores socio-ambientales de acuerdo con las recomendaciones de Munné et al. (2003).

\section{Evaluación de la diversidad de especies}

Se propuso realizar un análisis de la biodiversidad a nivel de especies (patrones de diversidad alfa), 
ya que permite dar cuenta del estado sistémico y funcional del ecosistema de las barrancas, con o sin impactos antrópicos evidentes. La estimación se realizó con los índices de Margalef $\left(D_{\mathrm{mg}}\right)$ y de Pielou $\left(J^{\prime}\right)$, mediante las ecuaciones 1 y 2 (Franco 1992, Magurran 2004). El índice de Margalef se basa en la riqueza específica y el de Pielou en la estructura de la comunidad (abundancia relativa de individuos, la cual determina la importancia de cada especie). Este último es un índice de equidad cuyo planteamiento teórico se sitúa en la uniformidad que tienen las especies en el sitio de estudio (Moreno 2001).

$$
\begin{aligned}
& D_{\mathrm{mg}}=S-1 / \ln (N) \\
& J^{\prime}=H^{\prime} / H_{\max } \\
& H^{\prime}=-\sum_{\mathrm{i}=1}^{\mathrm{s}} P i(\ln P i) \\
& P i=n i / N \\
& H_{\max }=\ln S
\end{aligned}
$$

donde $S$ es el número de especies (riqueza específica), $N$ es el número de todos los individuos de todas las especies, $P i$ es la proporción de individuos de la especie $i$ respecto al total de individuos $N$ (es decir la abundancia relativa de la especie $i$ ) y $n$ i es el número de individuos de la especie $i$ (Moreno 2001).

\section{Evaluación de la calidad de la barranca mediante un conjunto de indicadores socio-ambientales}

Esta propuesta se llevó a cabo para complementar la evaluación realizada con los índices de diversidad específica, en tanto que registra la condición de la cubierta vegetal de acuerdo con otras variables, a la vez que enfatiza el registro de las condiciones relacionadas con los impactos antrópicos y, por lo tanto, posibilita una evaluación más completa del estado ecosistémico de las barrancas. Se aplicaron nueve indicadores, la mayor parte tomados del Índice de Calidad del Bosque de Ribera (QBR) diseñado por Munné et al. (2003) para evaluar bosques riparios de ríos y arroyos, con las adaptaciones necesarias para los fines de este trabajo, y otros propuestos por nosotros:

- Calidad de la vegetación: conteo de individuos de especies alóctonas arbóreas y/o arbustivas para determinar si son individuos aislados o están conformando comunidades, dentro del área total de cada una de las barrancas.

- Grado de cubierta vegetal: porcentaje de área cubierta por árboles y/o arbustos respecto del área total.

- Estructura de la cubierta: porcentaje del área de terreno cubierta por sombra de árboles y/o arbustos respecto del área total.

- Continuidad con el sistema forestal adyacente: porcentaje del área colindante a la barranca en que el ecosistema de la barranca se mantiene.

- Continuidad de la comunidad a lo largo de la barranca: porcentaje de área dentro de la barranca donde en que la comunidad del bosque pierde continuidad, en función del área total.

- Grado de naturalidad de la barranca: tipo de modificaciones a la barranca como estructuras permanentes, modificación de paredes, formación de terraplenes, rellenado y/o canalización de las mismas.

- Actividades económicas dentro de la barranca: tipo y porcentaje del área comprometida por actividades económicas dentro de la barranca, como: pastoreo, cultivo, y extracción de materiales pétreos, entre otras.

- Presencia de casas- habitación: número de casashabitación dentro de la barranca o porcentaje del área de la barranca comprometida por construcciones, en función del área total.

- Contaminación de la barranca: tipo de residuos sólidos presentes en la barranca, así como de la presencia o no de aguas residuales.

El índice QBR (Munné et al. 2003) se basa en el análisis de cuatro componentes de igual peso específico ( 0 a 25 puntos) en la calificación final (0 a 100 Puntos): grado de cubierta vegetal riparia, Estructura de la cubierta, Calidad de la cubierta y alteraciones al cauce. Estos componentes pueden ser modificados por variables como Continuidad con el sistema forestal adyacente, Concentración de helofitos o arbustos en las orillas, Continuidad a lo largo de la barranca y Presencia de estructuras sólidas, entre otras.

En la propuesta de este trabajo se plantea como elemento vertebral el indicador compuesto Estado de la cubierta vegetal, dentro del cual se agrupa la mayor parte de los indicadores retomados de aquel trabajo con excepción de aquellos considerados no cruciales para el análisis de barrancas (e.g., Concentración de helófitos o arbustos en las orillas del cauce, debido a que las barrancas sólo presentan corrientes 
estacionales o únicamente corren en su cauce aguas residuales), en el entendido de que el ecosistema de las barrancas urbanas comparte algunas características con los bosques riparios, pero también difiere en otras.

Los cinco indicadores que se agrupan en el indicador compuesto y que evalúan aspectos de la flora son: Calidad de la vegetación, Grado de la cubierta vegetal, Estructura de la cubierta, Continuidad con el sistema forestal adyacente y Continuidad de la comunidad a lo largo de la barranca, de los cuales también se ha reconfigurado su ponderación. El indicador del índice QBR Grado de naturalidad del canal pluvial, se ha renombrado Grado de naturalidad de la barranca, con modificaciones en la variable que califica, las calificaciones que otorga y su peso dentro de la ponderación final. Se introducen en esta propuesta los indicadores Actividades económicas dentro de la barranca, Presencia de casas habitación y Contaminación de la barranca. Este último enfatiza la presencia de aguas residuales y residuos de manejo especial y peligrosos, mientras que Munné et al. (2003) sólo consideran la presencia de basura como un elemento que puede o no modificar al indicador Grado de naturalidad del canal pluvial.

La calificación que otorga a las barrancas el indicador compuesto se obtiene de la siguiente manera: el indicador simple Calidad de la vegetación aporta una calificación a la fracción de barranca correspondiente que puede ser de $0,10,20,30$ o 40 puntos. Los otros cuatro indicadores (Grado de la cubierta vegetal, Estructura de la cubierta, Continuidad con el sistema forestal adyacente y Continuidad de la comunidad a lo largo de la barranca) pueden o no modificar la calificación base aportada por el indicador Calidad de la vegetación. Las modificaciones pueden hacerse en puntajes de $-10,-5,+5 \mathrm{y}+10$. El resultado final para cada fracción evaluada no puede ser mayor de 40 puntos ni menor de 0 . La calificación total para el indicador compuesto Estado de la cubierta vegetal es la media aritmética de los valores de las fracciones evaluadas.

En esta propuesta, el valor global ( 0 a 100 puntos) es la suma simple de los puntajes aportados por los indicadores para el total de fracciones evaluadas en las que se haya dividido la barranca. El indicador compuesto Estado de la cubierta vegetal aporta de 0 a 40 puntos máximos a la calificación final, y su valor se suma con los otros cuatro indicadores: Grado de naturalidad de la barranca, Actividades económicas dentro de la barranca, Presencia de casas habitación y Contaminación de la barranca. Los dos primeros aportan de 0 a 10 puntos y los segundos de 0 a 20 puntos.
Se propone también una escala para los diversos niveles de calidad que pueden presentar las barrancas, la cual se muestra en el cuadro I.

CUADRO I. NIVELES DE CALIDAD.

\begin{tabular}{cc}
\hline Calidad & Puntuaciones \\
\hline Excelente & $\geq 90$ \\
Buena & $70-89$ \\
Regular & $50-69$ \\
Pobre & $30-49$ \\
Mala & $\leq 29$ \\
\hline
\end{tabular}

Fuente: elaboración propia con base en Munne et al. (2003).

\section{RESULTADOS Y DISCUSIÓN}

En los encinares de ambas barrancas se registraron 105 especies que pertenecen a 81 géneros y 40 familias de plantas vasculares. Se reconocieron ocho especies de encinos: Quercus acutifolia Née, Quercus castanea Née, Quercus crassipes Bonpl., Quercus desertícola Trel., Quercus glaucoides M. Martens \& Galeotti, Quercus laurina Bonpl., Querqus mexicana Bonpl. y Quercus rugosa Née., así como otros árboles (Salix bonplandiana Kunth, Juniperus flaccida Schltdl y Prunus salicifolia Kunth); 58 especies de herbáceas pertenecientes a diversos géneros entre los que destacan, por su número de especies, Agave (tres), Commelina (tres) e Ipomoea (cinco), y 26 especies de arbustos de las cuales destacan, por su abundancia de individuos en la zona de estudio, las pertenecientes a los géneros Acacia (2) y Rhus (2). Se registraron dos especies listadas en la NOM-059-SEMARNAT-2010 en la categoría de amenazadas: Dasylirion acrotrichum (Schiede) Zucc. y Erythrina coralloides DC. (SEMARNAT 2010).

Particularmente en la barranca El Conde, debido a la actividad silvícola de quienes habitan en la misma, se observó la introducción de especies no propias del ecosistema, como herbáceas (Cenchrus ciliaris L., Cynodon dactylon (L.) Pers.), arbustos (Nicotiana glauca Graham, Ricinus communis L.) y árboles (Eucalyptus globulus Labill., Populus alba L.)

La composición y riqueza del bosque de las barrancas demuestra, en concordancia con lo señalado por Vibrans (1997) “...que aun ambientes muy alterados en México pueden poseer una diversidad biológica considerable", lo cual se evidencia en las ocho especies de Quercus registradas para ambas barrancas y que se presentan en su totalidad en la barranca El 
Conde. Esto incluso si se compara con reportes sobre riqueza de fagáceas en bosques naturales de encino ya que dadas las dimensiones del sitio de estudio y las condiciones de deterioro ambiental de la barranca el Conde, su riqueza se les acerca e incluso en un caso le sobrepasa (Zacarías-Eslava y Del Castillo 2010, Sabás-Rosales et al. 2015, Mora-Donjuán et al. 2017, Martínez.-Meléndez y López-Santiago 2019).

\section{Evaluación con los índices de diversidad de es- pecies}

Los resultados arrojados mediante la aplicación de índices de diversidad de especies para las dos barrancas, se muestran en el cuadro II. De acuerdo con el índice de Margalef, el valor de 4.18 para la barranca El Conde indica que la misma presenta una mayor riqueza específica que la barranca Malinalli, cuyo valor es de 2.35. Dichos valores muestran que la diversidad de especies en ambas comunidades tiene un nivel medio, ya que valores inferiores a 2 indican baja diversidad y superiores a 5 denotan alta diversidad (Franco 1992, Magurran 2004). Esta interpretación concuerda con la lectura que Mora-Don Juan et al. (2017) hacen del valor de 0.18 para bosques de encino de la Sierra Madre del Sur, como de muy baja riqueza específica.

CUADRO II. ÍNDICES DE DIVERSIDAD DE ESPECIES.

\begin{tabular}{lcc}
\hline & Barranca Malinalli & Barranca El Conde \\
\hline Índice de Margalef & 2.35 & 4.18 \\
Índice de Pielou & 0.86 & 0.78 \\
\hline
\end{tabular}

En contraste, el índice de Pielou indica que la barranca El Conde es la menos diversa con un valor de 0.78 respecto a la barranca Malinalli con un valor de 0.86 . Esto, de acuerdo con la interpretación que se le da al índice de Pielou, en el cual magnitudes más pequeñas indican menor uniformidad en la población de cada especie. Es decir, que el valor 0 se refiere a la diversidad mínima y el 1 a la diversidad máxima (Moreno 2001). Conforme a los valores arrojados por este índice para ambas barrancas, se infiere que la diversidad de especies es de media a alta, ya que están por encima de los citados por García et al. (2019) para bosques de pino y encino en Chihuahua ( 0.75 y 0.77$)$, cuya valoración es de diversidad alta.

La mayor riqueza específica de la barranca El Conde, de acuerdo con el Índice de Margalef, podría explicarse por la actividad silvícola en el sitio con la introducción de especies, lo cual no ha significado la depredación total del ecosistema originario, ya que éste permanece y su diversidad se ha incrementado con las especies alóctonas introducidas. Lo anterior indica que seguramente los habitantes de la barranca encuentran útil el bosque originario, de ahí que no lo hayan sustituido totalmente por especies alóctonas, pues es claro que la conservación de los ecosistemas está estrechamente relacionada con la utilidad que los habitantes obtienen de ellos (Toledo y BarreraBassols 2008).

Por su parte, el índice de Pielou indica que la barranca El Conde presenta una mayor dominancia en su comunidad respecto a la barranca Malinalli, lo cual es propio de ecosistemas con intervención humana. Estos resultados aparentemente contradictorios se explican en tanto que evalúan la biodiversidad desde enfoques teóricos distintos, al ser el primero un índice que se basa en la riqueza de especies del sitio y el segundo una medida de la uniformidad de especies en el lugar.

El análisis de la diversidad específica de la flora de las barrancas permite observar un ecosistema (incluso en la barranca El Conde) en condiciones de estabilidad, en concordancia con Lädhe et al. (1999), quienes afirman que dicho análisis es importante como herramienta para evaluar la estabilidad de un bosque.

\section{Evaluación mediante indicadores socio-ambien- tales}

Los resultados de la aplicación del indicador compuesto para ambas barrancas se presentan en el cuadro III y los resultados de la aplicación de los otros cuatro indicadores, agrupados con la denominación Indicadores de impacto antrópico, se presentan en el cuadro IV.

El resultado del indicador compuesto Estado de la cubierta vegetal para la barranca Malinalli fue de 40 puntos contra 18.8 puntos para la barranca El Conde. Esto se explica cuando se observa que, al momento de evaluar las barrancas con el primer indicador simple, Calidad de la vegetación, los resultados son bajos para la barranca El Conde respecto a la barranca Malinalli, ya que la primera es abundante en especies alóctonas. Respecto al segundo indicador simple, Grado de cubierta vegetal, la puntuación disminuye aún más para la barranca El Conde, en la medida en que presenta áreas desmontadas tanto por la construcción de casas habitación como por las actividades de pastoreo. Situación similar se presenta con el indicador Estructura de la cubierta vegetal, ya que la barranca El Conde ha perdido árboles en detrimento de arbustos en algunas áreas, o bien ambos componentes por las razones arriba expuestas. Con la misma tendencia, los indicadores Continuidad 
a. Estado de la cubierta vegetal: 40 puntos

$i$. Calidad de la vegetación

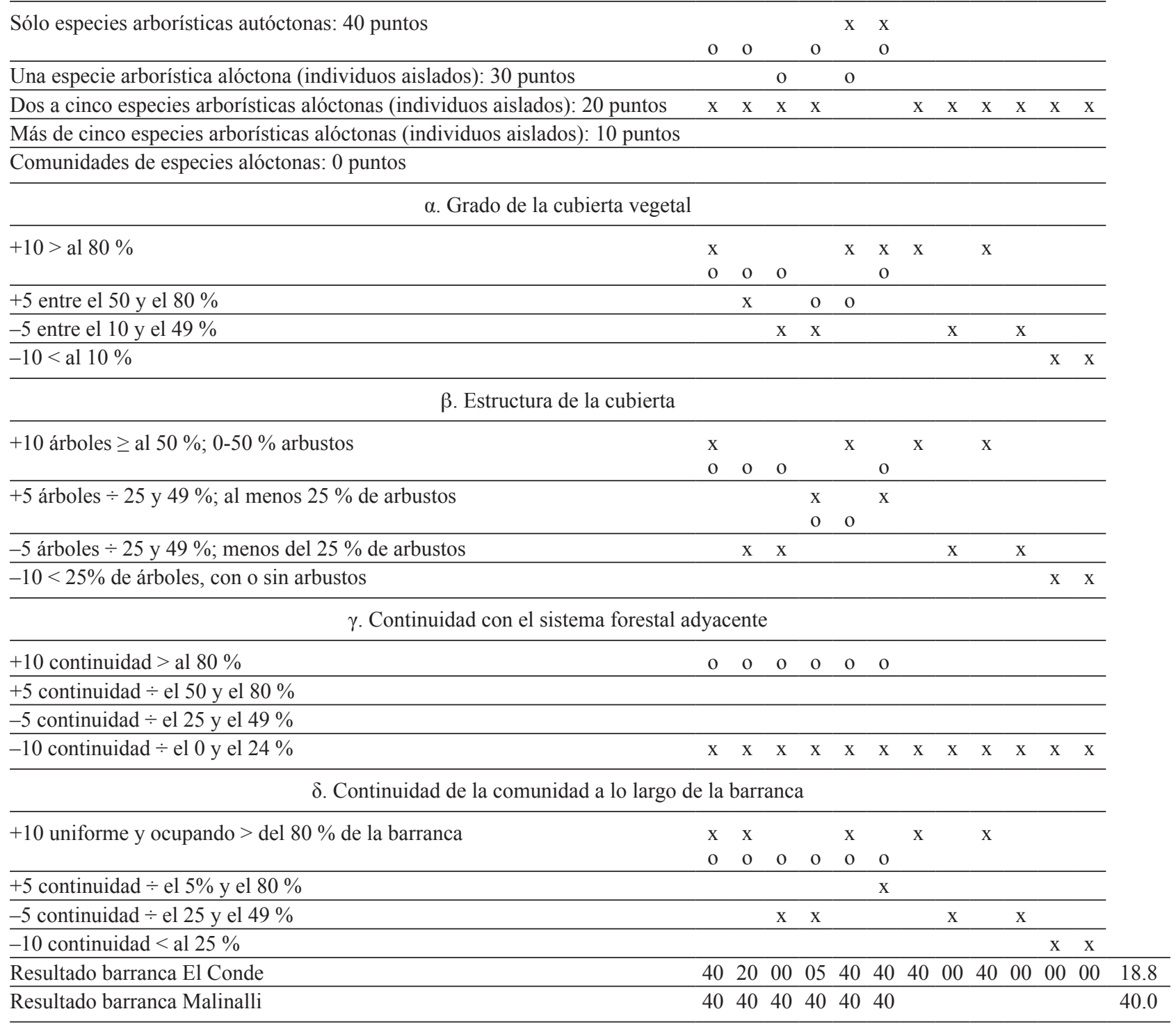

x: barranca El Conde, o: barranca Malinalli.

Fuente: Elaboración propia con base en Munne et al. (2003).

con el sistema forestal adyacente y Continuidad de la comunidad a lo largo de la barranca, marcan bajas puntuaciones para la barranca El Conde, ya que el ecosistema ha quedado aislado por el cambio de uso de suelo en sus alrededores sin que existan zonas de amortiguamiento a las orillas de la barranca. Además, dicho ecosistema se ha visto fragmentado por las casas habitación presentes, por la habilitación de una calle dentro de la misma, por las zonas para pastoreo, e incluso por pequeños huertos familiares.

Respecto a los otros cuatro indicadores, las puntuaciones son también muy bajas para la barranca El Conde, ya que en la misma existen estructuras de cemento para canalizar el agua, tubos de desagüe, vados, actividades de pastoreo, pequeños sembradíos, casas habitación y contaminación por residuos 
CUADRO IV. CALIFICACIONES APORTADAS POR LOS INDICADORES DE IMPACTO ANTRÓPICO.

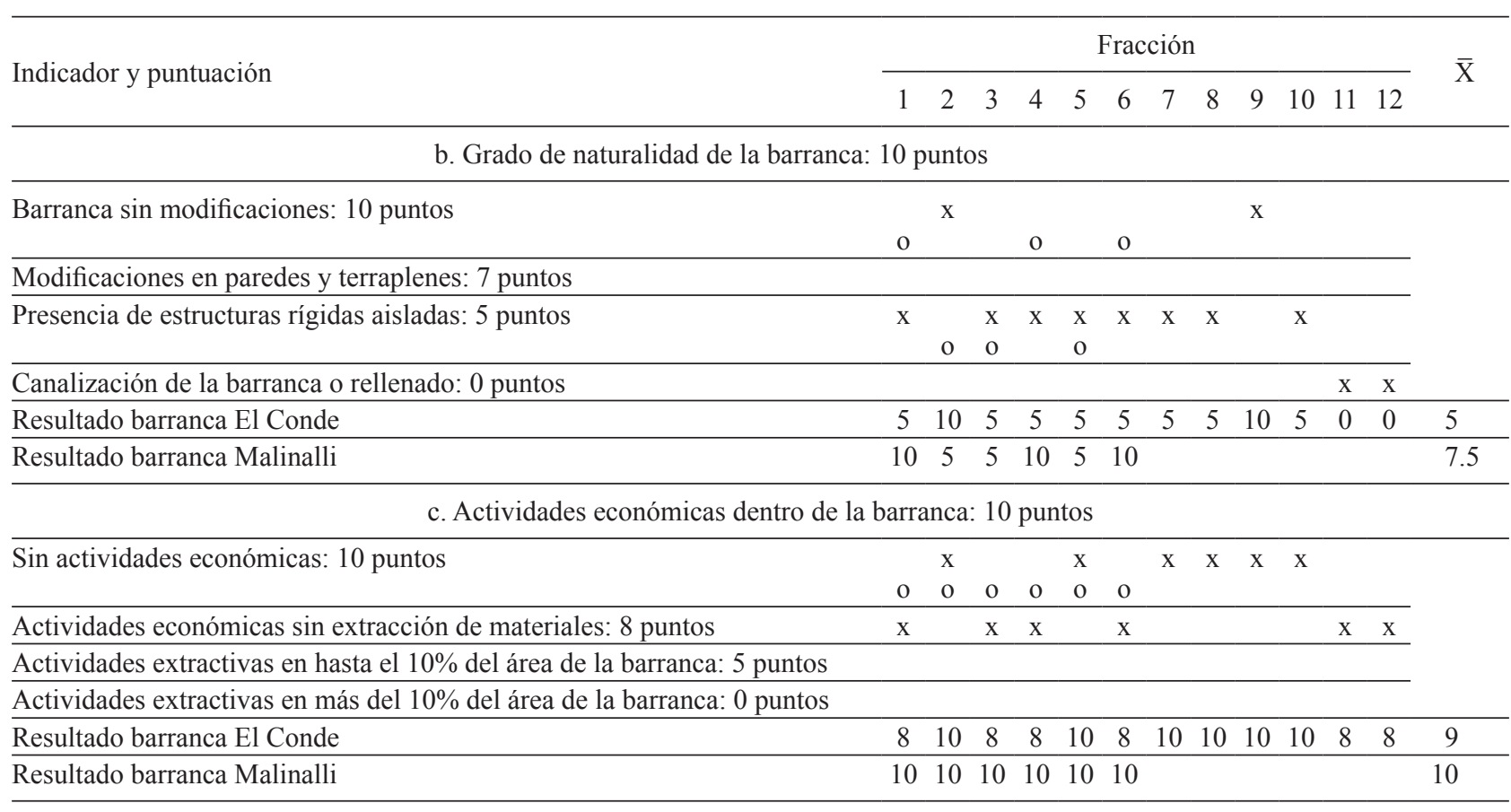

d. Presencia de casas habitación: 20 puntos

Sin casas habitación: 20 puntos

Una casa-habitación: 15 puntos

\begin{tabular}{lllllllll}
$x$ & & $x$ & & $x$ & & $x$ & $x$ & \\
0 & 0 & 0 & 0 & 0 & 0 & & & \\
\hline & & $x$ & & $x$ & & & \\
\hline
\end{tabular}

Núcleo de 2 a 10 casas habitación: 10 puntos

x $\quad x \quad$ x $\quad x$

Núcleo de 11 casas hasta un $49 \%$ del área de la barranca: 5 puntos

Núcleo habitacional en un área igual o mayor al $\mathrm{x}$

$50 \%$ del área de la barranca: 0 puntos

Resultado barranca El Conde

Resultado barranca Malinalli

$\begin{array}{lllllllllllll}20 & 10 & 20 & 15 & 20 & 15 & 20 & 10 & 20 & 10 & 10 & 0 & 14.1 \\ 20 & 20 & 20 & 20 & 20 & 20 & & & & & & & 20\end{array}$

e. Contaminación de la barranca: 20 puntos

Sin contaminación de agua: 20 puntos

$\begin{array}{llllll}0 & 0 & 0 & 0 & 0 & 0\end{array}$

Agua contaminada c/s residuos sólidos: 0 puntos

\begin{tabular}{llllllllllll}
$\mathrm{x}$ & $\mathrm{x}$ & $\mathrm{x}$ & $\mathrm{x}$ & $\mathrm{x}$ & $\mathrm{x}$ & $\mathrm{x}$ & $\mathrm{x}$ & $\mathrm{x}$ & $\mathrm{x}$ & $\mathrm{x}$ & $\mathrm{x}$ \\
\hline
\end{tabular}

Modificaciones a la primera puntuación

Presencia de residuos sólidos urbanos: -5 puntos

$\begin{array}{llllllllllll}\mathrm{x} & \mathrm{x} & \mathrm{x} & \mathrm{x} & \mathrm{X} & \mathrm{x} & \mathrm{x} & \mathrm{x} & \mathrm{x} & \mathrm{x} & \mathrm{x} & \mathrm{x}\end{array}$

Presencia de residuos de manejo especial: -10 puntos

o $\quad 0$

$\begin{array}{llllllllllll}x & x & x & x & x & x & x & x & x & x & x & x\end{array}$

o $\quad 0$

Presencia de residuos peligrosos: -15 puntos

Resultado barranca El Conde

$\begin{array}{rcccccccccccr}0 & 0 & 0 & 0 & 0 & 0 & 0 & 0 & 0 & 0 & 0 & 0 & 0 \\ 5 & 5 & 20 & 20 & 20 & 20 & & & & & & & 15\end{array}$

Resultado barranca Malinalli

x: barranca El Conde, o: barranca Malinalli.

Fuente: Elaboración propia con base en Munne et al. (2003).

sólidos domésticos y de manejo especial como cascajo y llantas, así como aguas residuales domésticas e industriales. En contraste, en la barranca Malinalli sólo se observó una cerca de alambre en una sección, así como algunos residuos sólidos domésticos como bolsas de plástico y dos residuos de manejo especial en la forma de llantas abandonadas.

En el cuadro $\mathbf{V}$ se muestran las puntuaciones totales para las barrancas, que corresponden a una calidad pobre para la barranca El Conde y excelente 
para la barranca Malinalli. Estos resultados permiten conocer, de manera general, el estado ecosistémico de las barrancas. En el caso de la barranca El Conde, el resultado pone en entredicho su continuidad al encontrarse en grave deterioro y riesgo, lo cual podría derivar en una grave pérdida para la ciudad en tanto que aún es un activo ecológico importante dada su riqueza y nivel de diversidad específica.

\section{CUADRO V. PUNTUACIONES TOTALES.}

\begin{tabular}{lcc}
\hline Indicadores & El Conde & Malinalli \\
\hline Estado de la cubierta vegetal & 18.8 & 40 \\
Grado de naturalidad de la barranca & 5 & 7.5 \\
Actividades económicas & & \\
dentro de la barranca & 9 & 10 \\
Presencia de casas habitación & 14.1 & 20 \\
Contaminación de la barranca & 0 & 15 \\
Total & 46.9 & 92.5 \\
\hline
\end{tabular}

Dichos resultados también permiten determinar en qué elementos particulares se experimentan los mayores impactos y con ello dirigir hacia ellos los esfuerzos de recuperación y conservación. De acuerdo con lo anterior, los impactos en la barranca El Conde son múltiples y los esfuerzos requieren de un programa integral de recuperación que abarque a los múltiples actores que participan en su degradación. Por su parte, en la barranca Malinalli los impactos son muy puntuales, por lo que las acciones podrían ser muy específicas.

Como ejercicio comparativo, la evaluación de las barrancas con el índice QBR hubiese arrojado un valor de 42 puntos para la barranca El Conde y 87.5 para la barranca Malinalli, que corresponden a una mala calidad (alteraciones fuertes) en el primer caso y a una buena calidad (bosque ligeramente perturbado) en el segundo (Munné et al. 2003). Estos resultados contrastan con los del indicador compuesto de la propuesta de este trabajo: Estado de la cubierta vegetal (18.8 y 40 puntos de 40 máximos, respectivamente, equivalentes a 47 y 100 puntos en una escala de 0 a 100 puntos). La calificación otorgada por este indicador compuesto es superior para ambas barrancas, sobre todo en Malinalli, en la medida en que sólo califica aspectos de la cubierta vegetal, mientras que el índice QBR incluye variables como presencia de estructuras rígidas y basura que modifica algunos de sus cuatro componentes.

En la propuesta de este trabajo, dichas variables se evalúan como indicadores independientes y permiten registrar particularidades, como en lo relativo a basura, aguas contaminadas y diferentes tipos de residuos sólidos, presentes comúnmente en barrancas urbanas. Los resultados del índice QBR también contrastan con los totales de los indicadores socio-ambientales (46.9 y 92.5, respectivamente). Estas diferencias muestran la importancia que adquieren dentro de la evaluación general los indicadores introducidos en la propuesta, que disminuyen el valor de la cubierta vegetal en el contexto general de la evaluación de la barranca y tienden a equilibrar el peso de los diferentes componentes en la ponderación final.

La escasa atención que se ha dado a las barrancas de la ciudad de Puebla - e incluso de otras ciudades más preocupadas por este tema- en trabajos académicos ha motivado la ausencia de reportes científicos sobre la condición ecosistémica de éstas. Sólo algunos reportes gubernamentales y algunas tesis presentan los resultados de estudios enfocados a algún componente de las barrancas, generalmente el análisis de la calidad del agua (Díaz 2014, García 2014) o recomendaciones sobre el manejo de las barrancas urbanas que generalmente derivan en la "necesidad" de entubarlas o canalizarlas y habilitar vialidades encima o a un lado de ellas (BUAP 2016). Esto concuerda con lo señalado por Munné et al. (2003), quienes plantean la necesidad de establecer una metodología de evaluación del estado de conservación del hábitat ripario, en un momento en que las mediciones de la calidad de agua eran la herramienta más usada para este fin, con sus limitaciones propias dado el alcance de sus conclusiones. Lo mismo ocurre actualmente en relación con las barrancas de Puebla.

La búsqueda infructuosa de una herramienta metodológica para evaluar barrancas ha incluido valoraciones de cuerpos de agua lénticos en zonas rurales de México (Rodríguez-Téllez et al. 2016) y de otras partes del mundo (López-Delgado et al. 2015), que dan cuenta del uso de la metodología de Munné et al. (2003), situación que motivó su consideración como base para la propuesta de una metodología para barrancas urbanas.

Los resultados de los índices de diversidad de especies y los indicadores socio-ambientales muestran que su complementariedad es viable y necesaria. Esto es evidente sobre todo para la barranca El Conde, en el entendido de que los indicadores socio-ambientales, que evalúan aspectos relacionados con la cubierta vegetal, registran aspectos que los indicadores de diversidad no consideran y que son importantes para determinar la calidad de la vegetación, y en consecuencia las estrategias para conservar el ecosistema original. Asimismo, los cuatro indicadores socioeconómicos muestran su pertinencia, en tanto que permiten 
valorar la participación de las variables evaluadas en el sistema, y con ello contemplarlas en un eventual programa de manejo de la barranca El Conde.

La propuesta de los indicadores socio-ambientales podría, en futuros trabajos, ampliarse hacia una gama de indicadores que contemplen variables no consideradas en este trabajo. Podría profundizarse en la consideración de las barrancas por su valor intrínseco —además de los servicios ecosistémicos que proveen al colectivo social - y ampliar la evaluación hacia aspectos relacionados con sus elementos simbólicos, su existencia misma y su legado (Linares y Romero 2008, Rodríguez y Cubillos 2012). Estos valores no se perciben de forma directa e inmediata, pero merecen tomarse en cuenta en el estudio de los bosques de barrancas urbanas, los cuales, por su naturaleza y composición florística, deben conservarse dadas sus características sui generis en un espacio altamente degradado.

\section{CONCLUSIONES}

Una mayor calidad ecológica se deriva de una mayor diversidad, lo cual justifica la introducción del concepto de diversidad en la conservación de ecosistemas (Magurran 2004). Así, la presencia de las familias, los géneros y las especies registrados en las barrancas, así como los niveles de diversidad específica de media a alta para ambas barrancas, son indicativos de que a pesar del impacto antrópico experimentado por la barranca El Conde, la riqueza de los ecosistemas se mantiene. Esto conforme a la aseveración de que la diversidad de especies es uno de los dos criterios (el otro es la rareza de los taxa) considerados por los ecólogos de la conservación para la preservación de ecosistemas (Margules y Usher 1981, Norton 2014).

Los resultados de la sistematización y aplicación de los nueve indicadores socioambientales como herramienta para evaluar barrancas urbanas reflejan, de forma integral y objetiva, las condiciones ecosistémicas de las mismas; además, sus resultados se complementan con la evaluación de la composición, riqueza y diversidad específica.

De acuerdo con esta metodología, la calidad pobre de la barranca El Conde refleja los impactos antrópicos experimentados y los disturbios generados sobre dicho ecosistema. Por ello su atención es urgente, en el entendido de que es necesario considerar los procesos de disturbio para conservar la diversidad de especies y su dinámica como bosque de encino (Hobbs y Huenneke 1992), ya que de continuar la presión hacia los bosques de las barrancas, la degradación se acentuará, con la extinción local de algunas especies aún existentes. El impacto del disturbio y su evaluación es relevante en las estrategias de conservación, sobre todo en áreas densamente pobladas, con miras a que las políticas de conservación se orienten hacia un impacto antrópico de baja intensidad (Vetaas 1997).

El estado ecosistémico pobre de la barranca El Conde debido a los fuertes impactos antrópicos que experimenta, permiten distinguir que no es exclusivo, sino un ejemplo de lo que ocurre en la actualidad (con algunas variantes) en la mayor parte del sistema de barrancas de la ciudad de Puebla. La condición ecosistémica de dicha barranca refleja las graves condiciones de contaminación que experimenta en contravención de la normatividad mexicana vigente en materia de eliminación de residuos sólidos y aguas residuales. Asimismo, refleja la manera en que ecosistemas con un nivel de biodiversidad de mediano a alto pueden experimentar un fuerte deterioro en otros de sus componentes, incluida la propia cubierta vegetal. Sin embargo, también permite observar la manera en que espacios fuertemente impactados aún constituyen importantes sitios naturales dentro de las ciudades.

Se reconocen las limitaciones de este trabajo, en el entendido de que deberá continuarse para afinar los indicadores propuestos de tal manera que sean sensibles a las variables consideradas y que contemplen, en la mayor medida posible, la integralidad del sistema. Asimismo, los resultados para la barranca El Conde, que muestran sus niveles de deterioro, exigen que la evaluación se haga extensiva a otras barrancas, acción que urge en el ámbito poblano en aras de rescatar un activo importante de la ciudad.

\section{REFERENCIAS}

Alcaraz A.F.J. (1999). Manual de teoría y práctica de geobotánica. ICE Universidad de Murcia y Diego Marín, Murcia, España, 199 pp.

Ayuntamiento de la Ciudad de Puebla (2008). Plan municipal de desarrollo 2008-2011. Ayuntamiento de la Ciudad de Puebla, Puebla, México [en línea]. http:// implanpuebla.gob.mx/index.php/planes-proyectos-y-publicaciones/item/25-titulo-2011-2014 22/09/2019

BUAP (2016). Ingenieros de la BUAP estudian los riesgos de vivir cerca de barrancas. Benemérita Universidad Autónoma de Puebla [en línea]. https://www.buap.mx/ content/ingenieros-de-la-buap-estudian-los-riesgosde-vivir-cerca-de-barrancas 27/04/2020 
City of Toronto (2018). The Toronto ravine strategy. City of Toronto [en línea]. https://www.toronto.ca/city-government/accountability-operations-customer-service/ long-term-vision-plans-and-strategies/parks-forestryrecreation/ravine-strategy/30/08/2019

CONAPO (2018). Sistema urbano nacional y zonas metropolitanas. Consejo Nacional de Población, México [en línea]. https://www.gob.mx/conapo/acciones-yprogramas/sistema-urbano-nacional-y-zonas-metropolitanas 10/10/2019

Díaz L.A.E. (2014). Determinación de la calidad de aguas residuales vertidas al rio Atoyac de industrias ubicadas en los corredores industriales de la ciudad de Puebla. Tesis de Licenciatura. Facultad de Ingeniería Química, Benemérita Universidad Autónoma de Puebla, Puebla, México, 78 pp.

FAO (2019). Servicios ecosistémicos y biodiversidad. Food and Agriculture Organization [en línea]. http:// www.fao.org/ecosystem-services-biodiversity/es/ $08 / 09 / 2019$

Franco L.J. (1992). Manual de ecología. Editorial Trillas, México, 266 pp.

Gamboa O.L. (2010). Las actividades económicas. Negocios y negociantes en la ciudad de Puebla, 18101913. Ediciones de Educación y Cultura, Benemérita Universidad Autónoma de Puebla, Puebla, México, $219 \mathrm{pp}$.

García S.M.E. (2014). Determinación de metales pesados totales $\mathrm{Cd}, \mathrm{Cr}, \mathrm{Fe}, \mathrm{Ni}, \mathrm{Pb}$ y $\mathrm{Zn}$ en aguas residuales vertidas a las barrancas por dos parques industriales en la ciudad de Puebla, México. Tesis de Licenciatura. Facultad de Ingeniería Química, Benemérita Universidad Autónoma de Puebla, Puebla, México, 91 pp.

García G.S.A., Narváez F.R., Olivas G.J.M. y Hernández S.J. (2019). Diversidad y estructura vertical del bosque de pino-encino en Guadalupe y Calvo, Chihuahua. Rev. Mex. Cien. For. 10 (53), 41-63. https://doi. org/10.29298/rmcf.v10i53.173

Gutiérrez P.V. y Silva G.S.E. (2017). Las barrancas: cambio de marco epistémico y su revaloración. Revista Latinoamericana el Ambiente y las Ciencias 8 (17), 65-74.

Hobbs R.J. y Huenneke L.F. (1992). Disturbance, diversity and invasion: implications for conservation. Conserv. Biol. 6 (3), 324-337. https://doi.org/10.1046/j.15231739.1992.06030324.x

IG-UNAM (1989). Diccionario geomorfológico. Publicaciones UNAM, México, 479 pp.

IMTA-CONAGUA (2013). Estudio de clasificación del Rio Atoyac, Puebla-Tlaxcala, informe final, resumen ejecutivo, 2008. Instituto Mexicano de Tecnología del Agua-Comisión Nacional del Agua [en línea]. http://www.cofemermir.gob.mx/mir/uploadtests/1981

1.66.59.2.Resumen\%20Ejecutivo\%20ver\%2007\%20

Estudio\%20Declaratoria.pdf 10/09/2019

INEGI (1994). Estadísticas históricas de México. Instituto Nacional de Estadística, Geografía e Informática [en línea]. http://www.amhe.mx/afiliacion/candidatos/ alejandra-2015-06-11/EHM_4.pdf 22/09/2019

INEGI (2015). Censo de población y vivienda 2015. Instituto Nacional de Estadística, Geografía e Informática [en línea]. https://www.inegi.org.mx/programas/intercensal/2015/21/09/2019

Lähde E., Laiho O., Norokorpi Y. y Saksa T. (1999). Stand structure as the basis of diversity index. Forest Ecol. Manag. 115 (2-3), 213-220. https://doi.org/10.1016/ S0378-1127(98)00400-9

Linares L.P. y Romero L.C. (2008). Economía y medio ambiente: herramientas de valoración ambiental. En: Tratado de tributación medioambiental (Becker Z.F., Cazorla P.L.M. y Martínez-Simancas S.J., Coord.). Thomson Reuters Aranzadi Editores, España, 11891225.

López M.E. (2005). Urbanización y observatorios locales. Ciudades 68, 2-10.

López-Delgado E., Vásquez-Ramos J., Villa-Navarro F. y Reinoso-Florez G. (2015). Evaluación de la calidad del bosque de ribera, utilizando un método simple y rápido en dos ríos de bosque seco tropical (Tolima, Colombia). Revista Tumbaga 1 (10), 6-29.

Lovejoy T. (2002). Prefacio. En: Un enfoque en la naturaleza: evaluaciones ecológicas rápidas (Sayre R., Ed.). The Nature Conservancy, Arlington, Virginia, USA, xvii-xviii.

Magurran A.E. (2004). Measuring biological diversity. Blackwell Science, Oxford, UK, 256 pp.

Margules C.R y Usher M.B. (1981). Criteria used in assessing wildlife conservation potential: A review. Biol. Conserv. 21 (2), 79-109. https://doi.org/10.1016/00063207(81)90073-2

Martínez-Meléndez N. y López-Santiago J.W. (2019). Diversidad de encinos en un refugio de la Sierra Madre de Chiapas. Desde el Herbario CICY 11, 102-107.

Mora-Donjuán C.A., Burbano-Vargas O.N., MéndezOsorio C. y Castro-Rojas D.F. (2017). Evaluación de la biodiversidad y caracterización estructural de un Bosque de Encino (Quercus L.) en la Sierra Madre del Sur, México. Revista Forestal Mesoamericana Kurú, 14 (35), 68-75. https://doi.org/10.18845/rfmk. v14i35.3154

Moreno C.E. (2001). Métodos para medir la biodiversidad, vol.1. M y T-Manuales y Tesis, Zaragoza, España, 83 pp.

Munné A., Prat N., Solá C., Bonada N. y Rieradevall M. (2003). A simple field method for assessing the 
ecological quality of riparian habitat in rivers and streams: QBR index. Aquatic Conserv.-Mar. Freshw. Ecosyst. 13, 147-163. https://doi.org/10.1002/aqc.529 Norton B.G. (2014). The preservation of species. Princeton Universiy Press, Princeton, USA, 320 pp.

Nowak D., Crane D. y Stevens J. (2006). Air pollution removal by urban trees and shrubs in the United States. Urban For. Urban Green. 4 (3-4), 115-123. https://doi. org/10.1016/j.ufug.2006.01.007

Rivas T.D. (2001). Importancia y ambiente de los bosques y árboles urbanos. Universidad Autónoma Chapingo, Estado de México, México, 81 pp.

Rodríguez R.P.C. y Cubillos G.A. (2012). Elementos para la valoración integral de los recursos naturales: un puente entre la economía ambiental y la economía ecológica. Una revisión de bibliografía. Gestión y Ambiente 15 (1), 77-90.

Rodríguez-Téllez E., García-de Jalón D., Pérz-López M.E., Torres-Herrera S.I., Ortiz-Carrasco R., PompaGarcía M., Morales-Montes M., García-García D.A., Zamudi-Castillo Eréndira y Vásquez-Vásquez L. (2016). Caracterización de la calidad ecológica del bosque de galería del río La Sauceda, Durango, México. Hidrobiológica 26 (1), 35-40.

Sabás-Rosales J.L., Sosa-Ramírez J. y Luna-Ruiz J. (2015). Diversidad, distribución y caracterización básica del hábitat de los encinos (Quercus: Fagaceae) del estado de San Luis Potosí, México. Bot. Sci. 93 (4), 881-897. https://doi.org/10.17129/botsci.205

Salgado M.S.S. (2018). La lenta muerte de los ríos de la ciudad de Puebla. Saberes y Ciencias 74 [en linea]. https://saberesyciencias.com.mx/2018/04/09/la-lentamuerte-los-rios-la-ciudad-puebla/ 02/10/2019

Sayre R. (Ed.). (2002). Un enfoque en la naturaleza: evaluaciones ecológicas rápidas. The Nature Conservancy, Arlington, Virginia, USA, 196 pp.

SEDEMA (2000). Ley Ambiental de Protección a la Tierra en el Distrito Federal. Secretaría de Medio Ambiente de la Ciudad de México [en línea]. http://www.aldf.gob. mx/archivo-3cd1aa41964e3f9735705a55d1ba096e.pdf

SEMARNAT (1997). Norma Oficial Mexicana NOM001-SEMARNAT-1996 que establece los límites máximos permisibles de contaminantes en las descargas de aguas residuales en aguas y bienes nacionales. Secretaría de Medio Ambiente y Recursos Naturales. Diario Oficial de la Federación, 6 de enero.

SEMARNAT (2003). Ley General para la Prevención y Gestión Integral de los Residuos. Secretaría de Medio Ambiente y Recursos Naturales. Diario Oficial de la Federación, 8 de octubre.

SEMARNAT (2010). Norma Oficial Mexicana NOM059-SEMARNAT-2010. Protección ambiental. Especies nativas de México de flora y fauna silvestres. Categorías de riesgo y especificaciones para su inclusión, exclusión o cambio. Lista de especies en riesgo. Secretaría de Medio Ambiente y Recursos Naturales. Diario Oficial de la Federación, 6 de marzo.

Silva G.S.E., Bonilla F.M.N., Toxtle T.J.S. y Pérez A.R. (2014). Fauna feral y/o nociva en colonias, alrededor de dos parques industriales y tres barrancas, en Puebla, México. Revista Iberoamericana de Producción Académica y Gestión Educativa 02, 1-25.

SIMPPI (2018). Parques industriales en el Estado de Puebla. Sistema Mexicano de Promoción de Parques Industriales [en línea]. http://www.contactopyme. gob.mx/parques/PARGEO1.ASP?ESTADO $=21$ 25/09/2019

Toledo V.M. y Barrera-Bassols N. (2008). La memoria biocultural. La importancia ecológica de las sabidurías tradicionales. Junta de Andalucía/Icaria Editorial, Barcelona, España, 230 pp.

TPL (2013). Versión 1.1 [en línea]. http://www.theplantlist. org

Vetaas O.R. (1997). The effect of canopy disturbance on species richness in a central Himalaya oak forest. Plant Ecol. 132 (1), 29-38. https://doi. org/10.1023/A:1009751219823

Vibrans H. (1997). Lista florística comentada de plantas vasculares silvestres en San Juan Quetzalcoapan, Tlaxcala, México. Acta Bot. Mex. 38, 21-67.

Zacarías-Eslava Y. y del Castillo R.F. (2010). Comunidades vegetales templadas de la Sierra Juárez, Oaxaca: Pisos altitudinales y sus posibles implicaciones ante el cambio climático. Bol. Soc. Bot. Méx. 87, 13-28. 